\title{
Impact of anticoagulation therapy on outcomes in patients with cardiac implantable resynchronization devices undergoing transvenous lead extraction: A substudy of the ESC-EHRA EORP ELECTRa (European Lead Extraction ConTRolled) Registry
}

Regoli, François ; Auricchio, Angelo ; Di Cori, Andrea ; Segreti, Luca ; Blomström-Lunqvist, Carina ; Butter, Christian ; Deharo, Jean-Claude ; Kennergren, Charles ; Kutarski, Andrzej ; Laroche, Cecile ; Zalevskiy, Valery ; Luzzi, Giovanni ; Cano, Oscar ; Grabowski, Marcin ; Rinaldi, Christopher ; Bongiorni, Maria Grazia

\begin{abstract}
INTRODUCTION Little data are available on anticoagulation (AC) management in patients with cardiac resynchronization (CRT) devices who undergo transvenous lead extraction (TLE) procedure. We investigated the impact of AC on periprocedural complications in CRT patients undergoing TLE, enrolled in the ESC-EHRA European Lead Extraction ConTrolled (ELECTRa) registry. METHODS AND RESULTS All CRT patients treated with TLE enrolled in the registry were considered. Perioperative AC management was left to the discretion of the Center. Major and minor intraprocedural and postprocedural complications were compared between patients without AC (Gp1) and patients with AC (Gp2). Regression analyses were performed to identify predictors of complications for Gp2. Out of 734 CRT pts, 328 (44.7\%) were under AC (Gp2). Patients from Gp2 presented lower LVEF (Gp2 32.5 \pm 10.9 vs Gp1 $34.5 \pm 11.9 \%$; $\mathrm{P}=0.03$ ), more advanced heart failure disease (NYHA III/IV: Gp2 42.0 vs Gp1 $31.5 \% ; \mathrm{P}=0.02$ ), and renal impairment (Gp2 39.0 vs $\mathrm{Gp} 124.3 \%$; $\mathrm{P}<0.001$ ). Perioperative regimens included AC interruption (Gp2A: $\mathrm{n}=169,51.5 \%$ ), "bridging" (Gp2B: $\mathrm{n}=135,41.2 \%$ ), or continued AC (Gp2C: $\mathrm{n}=24,7.3 \%$ ). TLE complete success rates (98\% in both groups) and major complication rates were comparable for both groups; minor bleeding events were more frequent in Gp2 (5.5\%) compared to $\mathrm{Gp} 1(2.5 \%$; $\mathrm{P}=0.051)$. No independent predictors were identified for Gp2, but minor complications were associated with "bridging" approach (Gp2B: 16 events vs Gp2A/C: 9 events; P =0.020). CONCLUSION CRT patients treated with TLE under AC were more compromised but did not present more major complications compared to patients without AC. More minor complications were associated with "bridging" AC regimen.
\end{abstract}

DOI: https://doi.org/10.1111/jce.13946

Posted at the Zurich Open Repository and Archive, University of Zurich

ZORA URL: https://doi.org/10.5167/uzh-173219

Journal Article

Accepted Version

Originally published at:

Regoli, François; Auricchio, Angelo; Di Cori, Andrea; Segreti, Luca; Blomström-Lunqvist, Carina; Butter, Christian; Deharo, Jean-Claude; Kennergren, Charles; Kutarski, Andrzej; Laroche, Cecile; Zalevskiy, 
Valery; Luzzi, Giovanni; Cano, Oscar; Grabowski, Marcin; Rinaldi, Christopher; Bongiorni, Maria Grazia (2019). Impact of anticoagulation therapy on outcomes in patients with cardiac implantable resynchronization devices undergoing transvenous lead extraction: A substudy of the ESC-EHRA EORP ELECTRa (European Lead Extraction ConTRolled) Registry. Journal of cardiovascular electrophysiology, 30(7):1086-1095.

DOI: https://doi.org/10.1111/jce.13946 
François Regoli ORCID iD: 0000-0002-5936-4656

Impact of anti-coagulation therapy on outcomes in patients with cardiac implantable resynchronization devices undergoing transvenous lead extraction: a sub-study of the ESC-EHRA EORP ELECTRa (European Lead Extraction ConTRolled) Registry

F. Regoli ${ }^{1}, \mathrm{MD}, \mathrm{PhD}$, A. Auricchio ${ }^{1}, \mathrm{MD}, \mathrm{PhD}$, A. Di Cori $^{2}, \mathrm{MD}$, L. Segreti ${ }^{2}, \mathrm{MD}, \mathrm{C}$. Blomström-Lunqvist ${ }^{3}$, MD, PhD, C. Butter ${ }^{4}$, MD, JC. Deharo ${ }^{5}$, MD, C. Kennergren ${ }^{6}$, MD, A. Kutarski $^{7}, \mathrm{MD}, \mathrm{PhD}$, C. Laroche $^{8}$, V. Zalevskiy ${ }^{9}, \mathrm{MD}$, G. Luzzi ${ }^{10}, \mathrm{MD}$, O. Cano ${ }^{11}$, MD, M. Grabowski $^{12}$, MD, CA. Rinaldi ${ }^{13}$, MD, MG. Bongiorni ${ }^{2}$, MD, on behalf of the ESC-EHRA-EORP ELECTRa Registry Investigators

* A complete list of the ELECTRa Investigators is provided in the Appendix.

Affiliations: (1) Fondazione Cardiocentro Ticino, Lugano, Switzerland; (2) Azienda Ospedaliero-Universitaria Pisana, UO Cardiologia 2 SSN, Pisa, Italy; (3) Uppsala University, Department of Cardiology and Medical Science, Uppsala, Sweden; (4) Heart Center Brandenburg in Bernau/Berlin \& Brandenburg Medical School, Department of Cardiology, Ladeburger Straße 17, 16321 Bernau, Germany; (5) CHU La Timone, Cardiologie, Service du prof Deharo, 264 Rue Saint Pierre, 13385 Marseille, France; (6) Sahlgrenska University Hospital, Cardiothoracic Surgery, Sahlgrenska/SU, 41345 Goteborg, Sweden; (7) Department of Cardiology Medical University of Lublin, 8 Jaczewskiego St, 20-954 Lublin, Poland; (8) EURObservational Research Programme (EORP), European Society of Cardiology, 2035 routes des Colles, 06903 Sophia Antipolis, France; (9) DU Nationale Institute of CVS, Department of Arrhythmias, 6 M. Amosov st., 03680 Kiev, Ukraine; (10) A.O.U. Policlinico Consorziale di Bari, Piazza Giulio Cesare, 11, 70124 Bari, Italy; (11) Hospital Universitari I Politecnic La Fe, Electrophysiology Section, Av. Bulevar Sur, s/n, Planta 4 - Torre F, 46026 Valencia, Spain; (12) First Chair and Department of Cardiology, Medical University of Warsaw, ul. Banacha 1a, 02-097 Warsaw, Poland; (13) Cardiology Dept Guy's and St Thomas' Hospitals, London, UK SE1 7EH.

Short Title: Impact of anticoagulation on lead extraction in CRT patients

Conflicts of Interest: FR reports personal fees from Medtronic, Boston Scientific, LivaNova/Microport, Bayer, Abbott and Daichi Sankyo. outside the submitted work; AA reports personal fees from Medtronic, from Boston Scientific, from LivaNova, outside the submitted

This article has been accepted for publication and undergone full peer review but has not been through the copyediting, typesetting, pagination and proofreading process, which may lead to differences between this version and the Version of Record. Please cite this article as doi: 10.1111/jce.13946.

This article is protected by copyright. All rights reserved. 
work; CK reports personal fees from Medtronic, from Boston Scientific, and from Spectranetics/Philips, outside the submitted work; OC reports personal fees from Abbott, outside the submitted work. Other authors have nothing to disclose.

Funding: The following companies have supported the study: Medtronic, Cook Medical, Boston Scientific, Spectranetics and Zoll.

\title{
Corresponding author:
}

François Regoli, MD PhD

Division of Cardiology, Fondazione Cardiocentro Ticino

Via Tesserete 48, CH-6900 Lugano, Switzerland

Phone: +41918053351 - Fax: +41918053173

Email: francois.regoli@cardiocentro.org

\begin{abstract}
Introduction Little data are available on anticoagulation (AC) management in patients with cardiac resynchronization (CRT) devices who undergo lead extraction procedure (TLE). We investigated the impact of $\mathrm{AC}$ on peri-procedural complications in CRT patients undergoing TLE, enrolled in the ESC-EHRA European Lead Extraction ConTrolled (ELECTRa) registry.
\end{abstract}

Methods and Results All CRT patients treated with TLE enrolled in the registry were considered. Peri-operative AC management was left to the discretion of the Center. Major and minor intra- and post-procedural complications were compared between patients without AC (Gp1) and patients with AC (Gp2). Regression analyses were performed to identify predictors of complications for $\mathrm{Gp} 2$.

Out of 734 CRT pts, 328 (44.7\%) were under AC (Gp2). Patients from Gp2 presented lower LVEF (Gp2 32.5 \pm 10.9 vs Gp1 34.5 $\pm 11.9 \%$, p=0.03), more advanced HF disease (NYHA III/IV: Gp2 42.0 vs Gp1 31.5\%, p=0.02), and renal impairment (Gp2 39.0 vs Gp1 24.3\%, p<0.001). Perioperative regimens included AC interruption (Gp2A: n=169, 51.5\%), "bridging" (Gp2B: $\mathrm{n}=135,41.2 \%)$, or continued AC (Gp2C: $n=24,7.3 \%)$. TLE complete success rates $(98 \%$ in both groups) and major complication rates were comparable for both groups; minor bleeding events were more frequent in $\mathrm{Gp} 2$ (5.5\%) compared to $\mathrm{Gp} 1(2.5 \%, \mathrm{p}=0.051)$. No independent predictors This article is protected by copyright. All rights reserved. 
were identified for $\mathrm{Gp} 2$, but minor complications were associated with "bridging" approach (Gp2B: 16 events vs Gp2A/C: 9 events, $\mathrm{p}=0.020$ ).

Conclusion CRT patients treated with TLE under AC were more compromised, but did not present more major complications compared to patients without AC. More minor complications were associated with "bridging" AC regimen.

Key words: lead extraction complications; CRT and lead extraction; anticoagulation in lead extraction; resynchronization therapy and complications; CRT and lead management.

\section{Introduction}

Cardiac resynchronization therapy (CRT) is a well-established non-pharmacological therapy for patients with heart failure. Compared to other cardiac implantable electronic devices CIEDs, CRT is a more complex device (one additional lead), usually has a shorter battery longevity, and is indicated in clinically complex patients (1), leading to more frequent replacement and possibly to higher infection rate. Furthermore, about $70 \%$ of CRT patients is fitted with a device that includes an implantable cardioverter-defibrillator (CRT-D) (1), thus more prone to mechanical stress resulting in more frequent lead dysfunction and local infection compared to a CRT with pacing function only (CRT-P). Should one of the above-listed clinical and technical issues arise in a given CRT patient then removal of the lead and/or device becomes mandatory.

The recent European CRT Survey II (1) reported that at least $40 \%$ of CRT patients receive oral anticoagulants either with or without combination of oral antiplatelet therapy. Management of anticoagulation (AC) and/or antiplatelet therapy in CRT patients undergoing a transvenous lead extraction (TLE) has been investigated to a limited extent. Current recommendations by international scientific Societies are based on expert consensus and single experience from high-volume centers (2-4).

This article is protected by copyright. All rights reserved. 
The present study investigates the impact of anticoagulation therapy on intra- and periprocedural complications in CRT patients undergoing TLE and included in the prospectively designed, recently published ESC-EHRA ELECTRa registry (5).

\section{Methods}

\section{Study design and patient population}

The present study represents a post-hoc analysis of CRT patients who underwent TLE and were included in the ELECTRa registry. Details about patient recruitment and data management have been extensively presented in a previous publication (5). The executive committee in cooperation with the EURObservational Research Programme (EORP) provided the study design, protocol, and the scientific leadership of the registry under the responsibility of the EHRA Scientific Initiatives Committee. Ethics' committee approval of the protocol was obtained for all participating centers and all subjects enrolled gave written informed consent to participate in the study. The investigation conformed with the principles outlined in the Declaration of Helsinki.

\section{Study objectives}

The primary objective was the comparison of cumulative incidence and rates of major intraprocedural and post-procedural complications including deaths, between patients never treated with AC therapy (Group 1) and those who had AC therapy (Group 2) before TLE (Figure 1). This latter group of patients consisted of patients in whom AC was interrupted at TLE (Group 2A), patients in whom AC was maintained with low weight molecular heparin (LWMH) (Group 2B), and finally patients who maintained the same pre-operative AC (Group 2C). Secondary objectives included impact of AC therapy on minor complications, and the identification of specific predictors of any complication for Group 2.

Peri-procedural anticoagulation management

This article is protected by copyright. All rights reserved. 
Peri-procedural anticoagulation management was not predefined by the registry protocol but left to physician's discretion and center's standard practice. Anticoagulation therapy included direct vitamin $\mathrm{K}$ antagonists (VKA), novel oral anticoagulants (non-VKA) which included dabigatran, rivaroxaban, or apixaban, and fractionated/unfractionated heparin.

\section{Definitions}

Definitions published in the guidance documents by EHRA and by HRS were used to define procedural approaches, techniques, and outcomes have been extensively reported in the main manuscript (5). In brief, sheaths were classified as mechanical non-powered (polypropylene or similar plastic material) or powered (laser, radio-frequency electrosurgical or controlledrotational with threaded tip devices). TLE safety and efficacy was analyzed by considering the rate of procedure related complications (major and minor) and success/failures (radiological and clinical). A major complication was defined as the one related to the procedure that was life threatening or resulted in death, or any unexpected event that caused persistent or significant disability, or any event that required significant surgical intervention. A radiological failure was defined when more than a $4 \mathrm{~cm}$ length of a lead was abandoned after a removal attempt, partial success when less than a $4 \mathrm{~cm}$ of a lead remained in the patient body and complete success when the lead was completely removed. Clinical failure (considered for each patient) was defined when either a procedure related major complication or a failure to achieve the clinical outcome for which the TLE was scheduled occurred.

\section{Statistics}

Univariate analysis was applied to both continuous and categorical variables. Continuous variables were reported as mean \pm standard deviation $(\mathrm{SD})$ or as median and inter-quartile range (IQR). Among-group comparisons were made using a non-parametric test (Kruskal-Wallis test).

Categorical variables were reported as percentages (without missing values if applicable). Among-group comparisons were made using a Chi-square test or the Fisher's exact test. Comparative Kaplan Meier analysis was performed with Log Rank p determination to compute This article is protected by copyright. All rights reserved. 
cumulative incidences of major adverse events and deaths. A stepwise multiple logistic regression was used to determine the predictors of any complication for Group 2 (variables with $\mathrm{P}<0.05$ in univariate, except those with a high number of missing data). No interaction was tested. A two-sided p-value of 0.05 was considered as statistically significant. All the analyses were performed using SAS statistical software version 9.4 (SAS Institute, Inc., Cary, NC, USA).

\section{Results}

\section{Patient characteristics}

Out of the 3555 patients enrolled in the ESC-EHRA-EORP ELECTRa registry, 734 were CRT patients. Of these, 328 patients $(44.7 \%$, Group 2) were under chronic AC therapy, being the vast majority $(67.1 \%)$ on vitamin $\mathrm{K}$-antagonist (Figure 1). The demographic and clinical characteristics of Groups 1 and 2 are presented in Table 1, and were typical for a CRT patient cohort. Overall, there was a significant burden of co-morbidities represented by atrial fibrillation, diabetes, hypertension, renal and/or pulmonary insufficiency (Table 1). Importantly about $40 \%$ of the patients already experienced a previous lead or system revision. Patients who were on AC were older, had higher NYHA class, more frequently presented valvular heart disease and comorbidities (Table 1), but were however less treated with anti-platelet drugs. In contrast, indication to TLE, number of leads and dwelling time were similar between Groups 1 and 2); radiological success was nearly identical in the 2 main groups (Appendix, Table A-1).

\section{Peri-procedural anti-coagulation management}

For more than half of the CRT patients of Group 2 AC was interrupted (Group 2A, n=169, $51.5 \%$ ) with a median of 3 days (IQR 1-5 days), whereas AC interruption followed by "bridging" with fractionated (110 patients) or unfractionated heparin (25 patients) was performed in 135 patients (Group 2B). In 24 patients (7.3\%) TLE was performed under uninterrupted AC (Group 2C) (Figure 1).

This article is protected by copyright. All rights reserved. 
Group 2C patients more often presented underlying valvular heart disease (Group 2C: 45.8\%, Group 2B: $26.7 \%$, Group 2A: 22.9\%, p=0.057) and less often dilated cardiomyopathy (Group 2C 41.7\%, Group 2B 57.0\%, Group 2A 73.1\%, p<0.001). Furthermore, TLE indication was less often infective in Group 2C (Group 2C 50.0\%, Group 2B 72.6\%, Group 2A 66.3\%, $\mathrm{p}=0.0791$ ). Other characteristics between these 3 treatment groups are summarized in the Appendix, Table A-2.

\section{Primary objective}

A total of 29 major adverse events including 23 deaths occurred. The rates and cumulative incidences of the different major adverse events did not differ significantly between Groups 1 and 2 (Table 2). Major complication event rates for Groups 2 and 1 were respectively (Table 2): intraprocedural rates were $0.3 \%$ ( 1 event) and $0.7 \%(\mathrm{p}=0.427)$; post-procedural rates were $4.0 \%$ (13 events) and 3.0\% (12 events, $\mathrm{p}=0.454)$; rates of death were $3.7 \%$ ( $\mathrm{n}=12$ events) and $2.7 \%$ $(n=11$ events, $p=0.463)$. Cumulative incidences of death for any cause (Figure 2A) were 12.3\% (95\%CI 3.4-27.3\%) and 10.1\% (4.4-18.6\%, Log Rank $\mathrm{p}=0.703)$, and for any major complication (Figure 2B) were 15.5\% (95\%CI 5.4-30.6\%) and 13.9\% (6.5-24.3\%, Log Rank $\mathrm{p}=0.732$ ), for Groups 2 and 1, respectively.

Considering the different peri-operative AC treatment sub-groups, no differences were found in either the rates of intra- or postprocedural major complications or the rates of deaths. In the same way, cumulative incidences of any major complication or death did not differ between the three treatment groups (Figure 2C and 2D).

\section{Secondary objectives}

Even though the overall rate of minor complications did not differ between Group 2 (26 events, $7.9 \%$ ) and 1 ( 26 events, $6.4 \%, \mathrm{p}=0.470)$, minor bleeding events however occurred more frequently in Group 2 (Table 2). Bleeding events requiring blood transfusions in the postprocedural phase were significantly more frequently in Group 2 (6 events) compared to only 1

This article is protected by copyright. All rights reserved. 
event for Group $1(\mathrm{p}=0.028)$. Furthermore, surgical site hematoma events occurring mostly in the post-procedural phase were more frequent in Group 2 (8 events) compared to 3 events for Group 1 (3 events, $\mathrm{p}=0.059)$.

When considering the different AC therapy regimens, Group 2B presented a significant higher rate of overall minor complications compared to the other strategies (Group 2B: 16 events [11.9\%] vs Groups 2A/C: 9 events [4.7\%], $\mathrm{p}=0.020$ ). For Group 2B, all events occurred in the post-procedural phase. A greater number of venous thrombosis $(n=4)$, minor bleedings requiring blood transfusions ( $\mathrm{n}=5)$, and pocket hematoma occurred in this group $(\mathrm{n}=5)$, compared to Groups 2A/C (Table 3).

Factors associated with any intra- or post-procedural complications in Group 2 (40 events) identified at univariate analysis included TLE performed under general anesthesia (HR 3.78, 95\% CI 1.39-10.26, $\mathrm{p}=0.006)$ and laser-assisted technique (HR 2.36, 95\%CI 1.16-4.79, $\mathrm{p}=0.018$ ). NYHA class III revealed a trend in identifying patients at higher risk (HR 6.28, 95\%CI 0.81-48.71, $\mathrm{p}=0.065$ ) of complications. At multivariate analysis, no independent predictors of any complication were identified for Group 2.

\section{Discussion}

To our knowledge, this is the first and largest study aiming to evaluate the effect of AC management on intra-procedural and post-procedural complications of CRT patients undergoing TLE. Several novelties are presented, all of which may have significant clinical consequences. The TLE complication risk was not different between CRT patients with or without a history of AC. Minor bleeding events were however observed more frequently in AC patients, but did not result in an excess of major events. CRT patients can be considered a particularly fragile patient group because of their clinical profile represented by significant comorbidity burden, anatomical challenges due to multiple leads with long dwelling time. While no increase of overall risk of major potentially lethal intra-procedural complications compared to patients with IPGs or ICDs (Figure 3). The post-procedural phase following TLE in CRT patients was characterized by high This article is protected by copyright. All rights reserved. 
morbidity and mortality, regardless of whether the patients were under AC or not. Although these findings should be cautiously considered due to methodological limitations, these are reassuring as far as intra-procedural outcome is concerned, but warrant caution for post-procedural management. In considering different peri-procedural AC regimens, a significantly higher rate of minor post-procedural complications was observed when "bridging" strategy was performed. Avoiding this strategy is therefore advisable.

Effect of chronic anti-coagulation therapy on intra- and postprocedural complication after TLE in CRT patients

Anticoagulation indication in CRT patients is either stroke prevention of non-valvular atrial fibrillation, because of a prosthetic valve or, more rarely, due to deep venous thrombosis. TLE is considered a procedure which exposes patients to a high risk of major bleeding $(4,6)$. However, thus far data specifically addressing the impact of anticoagulation on TLE outcomes in high risk patients, such as heart failure patients with CRT devices, are lacking.

In the present study, despite the fact that CRT patients from the AC group were significantly more compromised in terms of heart failure disease and presented more frequently comorbidities, compared to their non-anticoagulated counterpart, major complications including deaths were comparable between the two groups. The rates of major intra-procedural and postprocedural complications as well as deaths between the 2 groups were similar and roughly $0.5 \%$, 3.5\%, and 3\% respectively. Regardless of whether CRT patients were treated with AC or not, the rates of post-procedural complications were remarkably higher than the rates detected for all CIED in the whole ELECTRa patient cohort (5) (Figure 3). The fact that a significantly greater proportion of no AC patients were treated with anti-platelet drugs may in part account for the lack of excess of adverse events for AC patients in spite of their greater clinical complexity.

Similar rates of major intra-procedural and peri-procedural complications (at one month) have been previously reported (7). In line with the present study, a smaller retrospective, multicenter experience (7) reported that localized bleeding, whether in the form of entry site This article is protected by copyright. All rights reserved. 
bleeding requiring blood transfusion or pocket hematoma, represented the most frequent complication. In fact, the role of anticoagulation in determining increased bleeding events has been demonstrated in both studies. The study by Regoli and colleagues (7) identified anticoagulation therapy as an independent predictor of adverse events during the follow-up. Consistent with this finding, the present ELECTRa registry data showed that chronically anticoagulated CRT patients presented an overall rate of minor bleeding events more than twofold $(5.6 \%)$ that of not anticoagulated patients $(2.5 \%)$.

Early pocket revision due to pocket hematoma significantly increases the risk of surgical site infection $(8,9)$, especially in patients with CRT-D devices $(10)$. Measures to reduce the likelihood of pocket hematoma, including the positioning of a pocket drainage tube and a compressive medication are warranted. For patients at high risk of CIED infection use of the antibiotic pouch (11) is advised.

\section{Optimization of perioperative management of anti-coagulation therapy in CIED patients undergoing lead extraction}

Until now, there is little evidence in support of a specific anti-coagulation strategy for chronically anti-coagulated patients undergoing a lead extraction procedure. The recent 2017 Task Force document concludes that “... periprocedural anticoagulation strategies should be considered on a case-by-case basis, after assessing the thromboembolic risk during unprotected periods" (2). As outlined by an expert consensus document (4), careful clinical evaluation stratifying thromboembolic and bleeding risks in each patient is mandatory before any TLE procedure. However, these recommendations are not based on specifically designed controlled trials, but rather on expert consensus derived from single-center experiences of high-volume centers (3). This consensus, considers sub-therapeutic or therapeutic "bridging" with fractionated/unfractionated heparin in patients at high thromboembolic risk as a standard AC management strategy.

This article is protected by copyright. All rights reserved. 
The present study did observe, for the first time, an excess of minor bleeding events and, more importantly, of minor thromboembolic events associated with "bridging" AC strategy in the setting of TLE. This finding, coupled to the already well-known association between "bridging" approach and pocket hematoma during CIED de novo implantation or change (12), further strengthens the concept that "bridging" approach should be avoided altogether in periprocedural CIED management.

In more than half of CRT patients under chronic anticoagulation, peri-procedural management approach consisted in interrupting the anti-coagulant three days before the procedure without "bridging". Patients treated in this manner more frequently presented dilated cardiomyopathy, an infective indication for TLE, in whom device reimplant was more often deferred to another hospitalization. In this group, one out of every five patients were anticoagulated with a non-VKA anticoagulant (Appendix, Table A-2). The favourable pharmacodynamics of these anticoagulants as well as the recent availability of agents capable of reversing their effect, offer the advantage of controlling bleeding risk, while, at the same time, shortening the time in which the patient remains unprotected. These agents are now the therapy of choice for stroke prevention in patients with non-valvular atrial fibrillation and no or mild to moderate renal impairment. Since TLE is considered a procedure involving a high risk of major bleeding, current recommendations indicate non-VKA interruption 48 hours before the intervention and reinitiation not before 24 hours after (13).

The few CRT patients treated according to the AC continued approach (Group 2C) presented several differences compared to the other 2 groups. These patients more often presented valvular heart disease, a non-infective indication for TLE, with device re-implantation performed during the same hospitalization (Appendix, Table A-2). Most of the CRT patients in this group are assumed to be those at highest thromboembolic risk. Although the number of patients treated through continued $\mathrm{AC}$ was limited, the overall incidences of major and minor complications were not higher compared to the other 2 groups. Similar findings have been recently reported by a large single-center experience (14). In this study, TLE was performed This article is protected by copyright. All rights reserved. 
during uninterrupted warfarin therapy in patients at high risk of thromboembolic events with any CIED (5\%, 62 patients of the entire consecutive cohort). TLE was highly successful and only one major complication was reported, namely femoral vein vascular tear needing surgical repair.

\section{Study limitations}

The present study is a retrospective analysis of data from the ELECTRa registry comparing early outcomes after TLE between CRT patients treated pre-operatively with anticoagulation and those not treated with anticoagulation. Some important differences between the groups, especially the fact that no AC patients were more often under anti-platelet drugs, and also the type of electrode extracted (the coronary sinus electrode was more often targeted for extraction in the $\mathrm{AC}$ group), the type of device reimplanted after extraction, may all have influenced the comparative analysis in a non-quantifiable way.

The ELECTRa registry captures AC treatment strategies dating back a few years. Quite differently from what is reflected from the present registry data, most CRT patients with atrial fibrillation considered for TLE procedure are anticoagulated today with non-VKA anticoagulants.

\section{Conclusion}

Despite the fact that anticoagulated CRT patients were more compromised and fragile, TLE efficacy, cumulative incidences of periprocedural major complications and deaths, were all comparable to non-anticoagulated patients. The more frequent post-procedural minor bleeding events in anticoagulated patients, were mainly associated with the "bridging" approach. Until prospective, controlled clinical data for non-VKA anticoagulants become available, careful periprocedural anticoagulation management avoiding "bridging" anticoagulation strategy should continue to be the standard of practice.

This article is protected by copyright. All rights reserved. 


\section{Acknowledgements}

EURObservational Research Programme (EORP) Oversight and Registry Executive Committees.

Data collection was conducted by the EORP department from the ESC by Myriam Glemot as Project Officer, Maryna Andarala as Data Manager. Overall activities were coordinated and supervised by Doctor Aldo P. Maggioni (EORP Scientific Coordinator).

All investigators are listed in the Supplemental Appendix 1.

\section{References}

1. Dickstein K, Normand C, Auricchio A et al. CRT Survey II: a European Society of Cardiology survey of cardiac resynchronization therapy in 11088 patients-who is doing what to whom and how? Eur J Heart Fail 2018; 20: 1039-1051.

2. Kusumoto FM, Schoenfeld MH, Wilkoff BL et al. 2017 HRS expert consensus statement on cardiovascular implantable electronic device lead management and extraction. Heart Rhythm 2017.

3. Bongiorni MG, Soldati E, Zucchelli G et al. Transvenous removal of pacing and implantable cardiac defibrillating leads using single sheath mechanical dilatation and multiple venous approaches: high success rate and safety in more than 2000 leads. European Heart Journal. 2008;29:2886-2893.

4. Zacà V, Marcucci R, Parodi G et al. Management of antithrombotic therapy in patients undergoing electrophysiological device surgery. Europace. 2015 Jun;17(6):840-54.

5. Bongiorni MG, Kennergren C, Butter C et al. The European Lead Extraction ConTRolled (ELECTRa) study: a European Heart Rhythm Association (EHRA) Registry of Transvenous Lead Extraction Outcomes. Eur Heart J 2017; 38 :2995-3005.

This article is protected by copyright. All rights reserved. 
6. Regoli F, D'Ambrosio G, Caputo ML et al. New-onset pericardial effusion during transvenous lead extraction: incidence, causative mechanisms, and associated factors. J Interv Card Electrophysiol. 2018 Apr;51(3):253-261.

7. Regoli F, Bongiorni MG, Rordorf R et al. High recurrence of device-related adverse events following transvenous lead extraction procedure in patients with cardiac resynchronization devices. Eur J Heart Fail. 2016.

8. Uslan DZ, Gleva MJ, Warren DK et al. Cardiovascular implantable electronic device replacement infections and prevention: results from the REPLACE Registry. Pacing Clin Electrophysiol 2012;35:81-7.

9. Essebag V, Healey JS, Ayala-Paredes F et al.. Strategy of continued vs interrupted novel oral anticoagulant at time of device surgery in patients with moderate to high risk of arterial thromboembolic events: The BRUISE CONTROL-2 trial. Am Heart J. 2016 Mar;173:102-7.

10. Mittal S, Shaw RE, Michel K, Palekar R, Arshad A et al. Cardiac implantable electronic device infections: incidence, risk factors, and the effect of the AigisRx antibacterial envelope. Heart Rhythm 2014; 11: 595-601.

11. Henrikson CA, Sohail MR, Acosta $\mathrm{H}$ et al. Antibacterial Envelope Is Associated With Low Infection Rates After Implantable Cardioverter-Defibrillator and Cardiac Resynchronization Therapy Device Replacement: Results of the Citadel and Centurion Studies. JACC Clin Electrophysiol. 2017 Oct;3(10):1158-1167.

12. Birnie DH, Healey JS, Wells GA, Verma A et al. Pacemaker or defibrillator surgery without interruption of anticoagulation. N Engl J Med 2013; 368: 2084-93.

13. Steffel J, Verhamme P, Potpara TS et al. ESC Scientific Document Group. The 2018 European Heart Rhythm Association Practical Guide on the use of non-vitamin K 
antagonist oral anticoagulants in patients with atrial fibrillation: executive summary. Europace. 2018; 20: 1231-1242.

14. Zheng Q, Maytin M, John RM, Killu AM, Epstein LM. Transvenous lead extraction during uninterrupted warfarin therapy: Feasibility and outcomes. Heart Rhythm 2018; 15: $1777-1781$.

15.

\section{Figures}

Figure 1. ELECTRa registry patient flow diagram presenting the proportion of CRT patients of the total enrolled patient cohort of the registry and the proportion of the CRT patients under chronic anticoagulation therapy. For patients treated with peri-operative anticoagulation (Group 2) details are provided on the anticoagulation agent chronically prescribed as well as the peri-operative anticoagulation strategies implemented, either anticoagulation interrupted without bridging (Group 2A), "bridging" strategy (Group 2 B), or continued anticoagulation (Group 2 C). IPG: implantable pulse generator; ICD: implantable cardioverter defibrillator; CRT-D/P: Cardiac resynchronization therapy defibrillator (D) or pacemaker (P); VKA: Vitamin K antagonist; LMWH: low-molecular weight heparin; NonVKA: Non-vitamin K antagonist.

Figure 1.

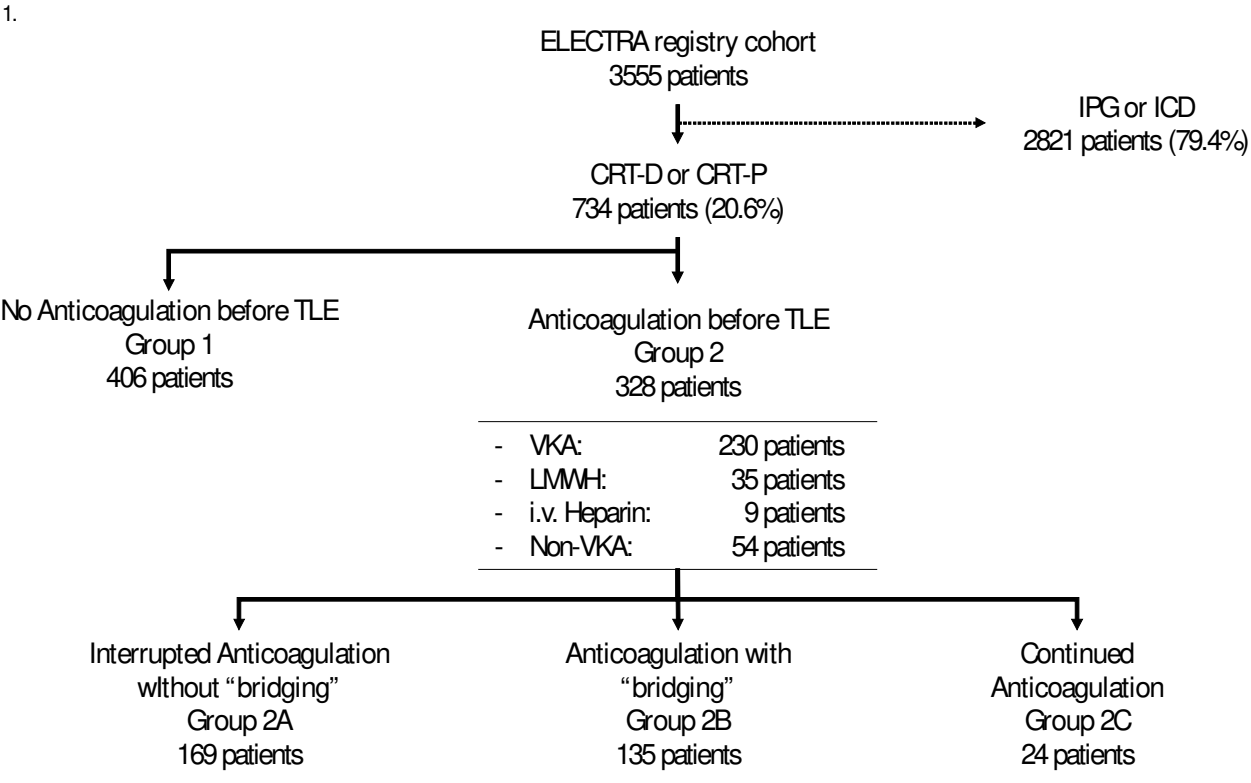

This article is protected by copyright. All rights reserved. 
Figure 2. Comparison of freedom from death for any cause after lead extraction between Group 1 (blue curve, no AC) and Group 2 (red curve, on AC), and between subgroups 2A (yellow, $\mathrm{AC}$ interrupted), 2B (red, $\mathrm{AC}$ bridging), and 2C (blue, $\mathrm{AC}$ continued) are presented in panels A and C, respectively. Comparison of freedom from major complications after lead extraction between Groups 1 and 2 and between subgroups 2A, 2B, and 2C are presented in panels B and D, respectively. Values are expressed as absolute and as percentage cumulative incidences with $95 \%$ confidence interval between parenthesis.

Figure 2.

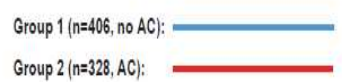

A

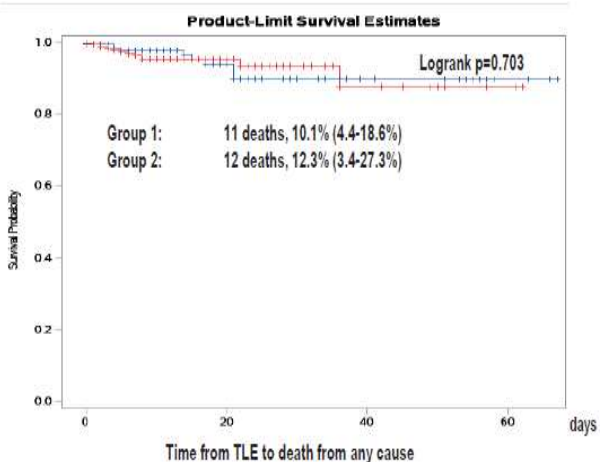

B

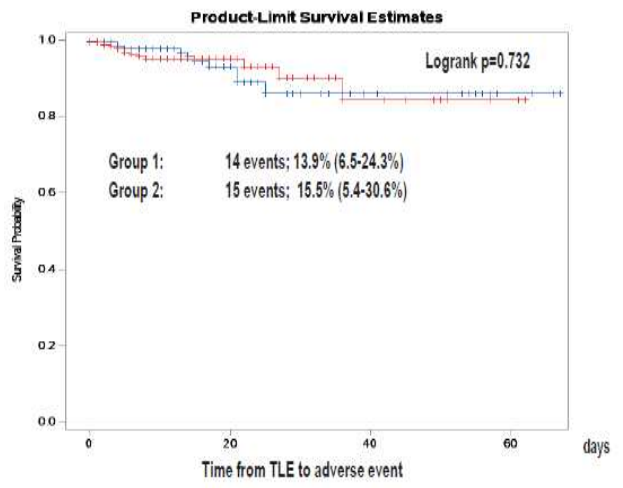

Group $2 \mathrm{~A}(\mathrm{n}=169, \mathrm{AC}$ interrupted):

Group $2 B$ ( $n=135, A C$ bridging):

Group $2 C$ ( $n=24, A C$ continued):

C

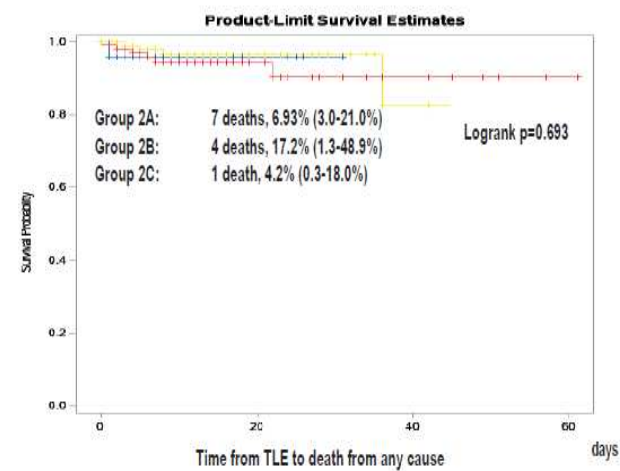

D

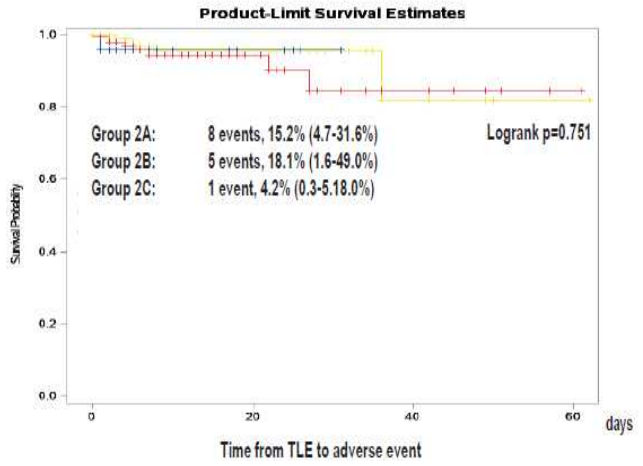

This article is protected by copyright. All rights reserved. 
Figure 3. Overall major and minor intra-procedural (Panel A) and post-procedural complication rates (Panel B) are presented for all groups, including the anticoagulation patient subgroups, 2A, 2B, and 2C. On the right of each panel corresponding overall intraprocedural (Panel A) and post-procedural (Panel B) complication rates of the complete ELECTRa registry cohort are presented (5).

Figure3.

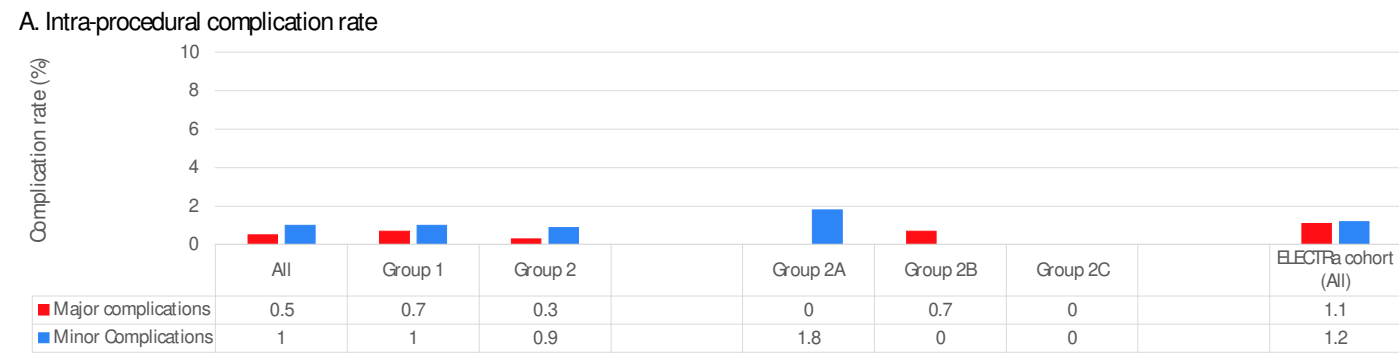

B. Post-procedural complication rate

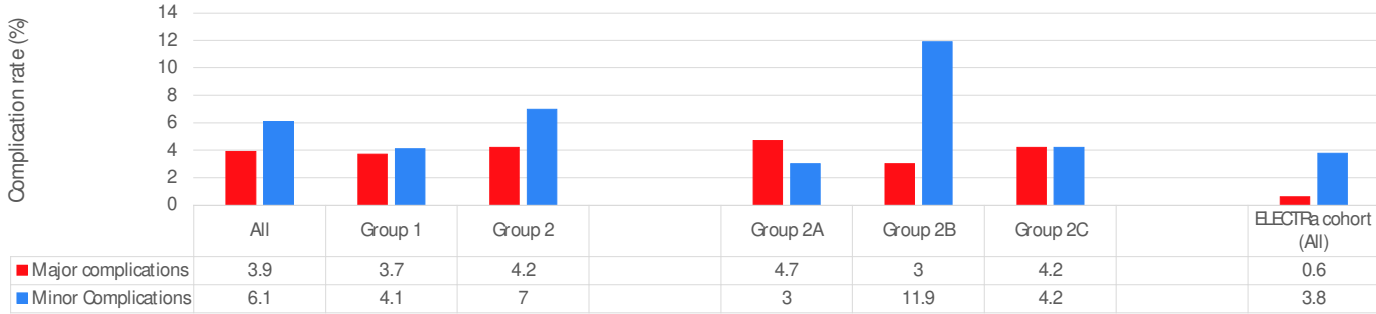

This article is protected by copyright. All rights reserved. 
Table 1. Demographic and peri-procedural characteristics.

\begin{tabular}{|c|c|c|c|c|c|}
\hline Variable & & Total $(N=734)$ & $\begin{array}{c}\text { Not treated with } \\
\text { AC }(\mathrm{N}=406) \\
\text { (Group 1) }\end{array}$ & $\begin{array}{c}\text { Treated with AC } \\
(\mathbf{N = 3 2 8 )} \\
(\text { Group 2) }\end{array}$ & p-value \\
\hline \multicolumn{6}{|l|}{ Demographic and clinical } \\
\hline Age (years) & & $68.1(11.3)$ & $67.4(11.5)$ & $68.9(11.1)$ & 0.091 \\
\hline Gender (Male) & Male & $602(82.0)$ & $328(80.7)$ & $274(83.5)$ & 0.335 \\
\hline Body Mass Index (kg/m2) & & $27.0(4.6)$ & $27.3(4.6)$ & $26.7(4.7)$ & 0.037 \\
\hline $\begin{array}{l}\text { Heart Disease Etiology } \\
\text { Coronary artery disease }\end{array}$ & & $361(49.2)$ & $192(47.5)$ & $169(52.3)$ & 0.198 \\
\hline Valvular heart disease & & $125(17.1)$ & $40(9.9)$ & $85(26.2)$ & $<0.001$ \\
\hline Dilated cardiomyopathy & & $495(67.7)$ & $286(70.6)$ & $209(64.1)$ & 0.061 \\
\hline Other heart disease & & $18(2.4)$ & $8(1.9)$ & $10(3.0)$ & 0.347 \\
\hline \multirow[t]{4}{*}{ NYHA } & I & $60(8.3)$ & $37(9.3)$ & $23(7.1)$ & 0.016 \\
\hline & II & $312(43.2)$ & $187(47.1)$ & $125(38.5)$ & \\
\hline & III & $246(34.1)$ & $122(30.7)$ & $124(38.2)$ & \\
\hline & IV & $20(2.7)$ & $6(1.5)$ & $14(4.3)$ & \\
\hline $\begin{array}{l}\text { Left Ventricular Ejection Fraction } \\
(\%)\end{array}$ & & 33.57 (11.5) & $34.45(11.9)$ & $32.49(10.9)$ & 0.029 \\
\hline Hypertension & & $433(59.8)$ & $243(60.7)$ & $190(58.6)$ & 0.565 \\
\hline Diabetes mellitus & & $247(34.1)$ & $137(34.5)$ & $110(33.7)$ & 0.829 \\
\hline Chronic kidney disease & & $226(30.9)$ & $98(24.3)$ & $128(39.0)$ & $<0.001$ \\
\hline $\begin{array}{l}\text { Chronic obstructive pulmonary } \\
\text { disease }\end{array}$ & & $91(12.4)$ & $49(12.1)$ & $42(12.8)$ & 0.780 \\
\hline \multicolumn{6}{|l|}{ Implanted device history } \\
\hline Device type CRT-pacemaker & & $127(17.3)$ & $69(17.0)$ & $58(17.6)$ & 0.806 \\
\hline CRT-defibrillator & & $607(82.7)$ & $337(83.0)$ & $270(82.3)$ & 0.806 \\
\hline Previous complications to CIED & & $254(34.6)$ & $146(35.9)$ & $108(32.9)$ & 0.390 \\
\hline \multirow[t]{4}{*}{ Previous system revisions } & 0 & $460(63.6)$ & $251(63.0)$ & $209(64.3)$ & 0.586 \\
\hline & 1 & $166(22.9)$ & $90(22.6)$ & $76(23.3)$ & \\
\hline & 2 & $55(7.6)$ & $32(8.0)$ & $25(7.7)$ & \\
\hline & $\geq 3$ & $40(5.5)$ & $25(6.3)$ & $15(4.6)$ & \\
\hline Previous attempt of lead extraction & & $30(4.1)$ & $19(4.7)$ & $11(3.4)$ & 0.366 \\
\hline \multicolumn{6}{|l|}{ Antiplatelet therapy } \\
\hline Aspirin & & $323(44.0)$ & $240(59.1)$ & $83(25.3)$ & $<0.001$ \\
\hline Clopidogrel & & $46(6.3)$ & $32(7.9)$ & $14(4.3)$ & 0.045 \\
\hline Prasugrel & & $5(0.7)$ & $4(1.0)$ & $1(0.3)$ & 0.265 \\
\hline Ticagrelor & & $1(0.1)$ & $1(0.2)$ & 0 & 0.368 \\
\hline Dual anti-platelet & & $28(3.8)$ & $22(5.4)$ & $6(1.8)$ & 0.012 \\
\hline Other & & $11(1.5)$ & $10(2.5)$ & $1(0.3)$ & $\mathbf{0 . 0 1 7}$ \\
\hline $\begin{array}{l}\text { Anticoagulation therapy } \\
\text { Interrupted }\end{array}$ & & $169(51.5)$ & NA & $169(51.5)$ & \\
\hline median [IQR], days & & $3.00[1.00-5.00]$ & NA & $3.00[1.00-5.00]$ & \\
\hline Bridging LMWH & & $101(74.8)$ & NA & $101(74.8)$ & \\
\hline Heparin iv. & & $25(18.5)$ & NA & $25(18.5)$ & \\
\hline Unknown & & $9(6.6)$ & NA & $9(6.6)$ & \\
\hline Continued & & $24(7.3)$ & NA & $24(100)$ & \\
\hline Medication Vitamin K antagonist & & $230(70.1)$ & NA & $230(70.1)$ & \\
\hline LMWH & & $35(10.7)$ & NA & $35(10.7)$ & \\
\hline Heparin & & $9(2.7)$ & NA & $9(2.7)$ & \\
\hline Non-VKA & & $54(16.5)$ & NA & $54(16.5)$ & \\
\hline
\end{tabular}

This article is protected by copyright. All rights reserved. 
Continuous variables are expressed as mean with corresponding standard deviation or median with corresponding IQR; categorical variables are expressed as absolute value with proportion between parenthesis. NYHA: New York Heart Association Class; LMWH: low molecular weight heparin; NA: not applicable; Non-VKA: Non-vitamin K-antagonist.

Table 2. Comparison of complications and deaths between CRT patients not treated with AC (Group 1) and treated with AC (Group 2).

\begin{tabular}{|c|c|c|c|c|}
\hline \multirow{2}{*}{ Variable } & \multirow{2}{*}{ Total $(N=734)$} & $\begin{array}{c}\text { Not treated } \\
\text { with } A C \\
(N=406)\end{array}$ & $\begin{array}{l}\text { Treated with } \\
\text { AC }(N=328)\end{array}$ & \multirow{2}{*}{ p-value } \\
\hline & & (Group 1) & (Group 2) & \\
\hline \multicolumn{5}{|c|}{ Intra- and post-procedural complications } \\
\hline MAJOR- total & $29(3.9)$ & $15(3.7)$ & $14(4.2)$ & 0.692 \\
\hline Intraprocedural & $4(0.5)$ & $3(0.7)$ & $1(0.3)$ & 0.427 \\
\hline Death & 2 & 2 & 0 & 0.203 \\
\hline Cardiac avulsion or tear & 1 & 1 & 0 & 0.368 \\
\hline Vascular avulsion or tear & 2 & 2 & 0 & 0.203 \\
\hline Respiratory or anesthesia & 1 & 0 & 1 & 0.261 \\
\hline Post-procedural & $25(3.4)$ & $12(3.0)$ & $13(4.0)$ & 0.454 \\
\hline Death & 21 & 9 & 12 & 0.244 \\
\hline Cardiac avulsion or tear & 2 & 1 & 1 & 0.880 \\
\hline Vascular avulsion or tear & 2 & 0 & 2 & 0.115 \\
\hline Stroke & 1 & 0 & 1 & 0.266 \\
\hline Respiratory or anesthesia & 2 & 2 & 0 & 0.203 \\
\hline
\end{tabular}

This article is protected by copyright. All rights reserved. 


\begin{tabular}{|c|c|c|c|c|}
\hline Total major bleeding & 7 & 4 & 3 & 1.000 \\
\hline Total major thromboembolic & 1 & 0 & 1 & 1.000 \\
\hline MINOR-total & $52(7.1)$ & $26(6.4)$ & $26(7.9)$ & 0.465 \\
\hline Intraprocedural & $7(1.0)$ & $4(1.0)$ & $3(0.9)$ & 0.922 \\
\hline Hematoma at surgical site req. reop. or drainage & 1 & 1 & 0 & 0.368 \\
\hline Blood transfusion & 3 & 1 & 2 & 0.443 \\
\hline Pulmonary embolism not req. surgery & 1 & 1 & 0 & 0.368 \\
\hline Arrythmias & 2 & 1 & 1 & 0.880 \\
\hline Post-procedural & $45(6.1)$ & $22(4.1)$ & $23(7.0)$ & 0.440 \\
\hline Pericardial effusion without drainage & 4 & 3 & 1 & 0.427 \\
\hline Hemotorax without chest tube & 1 & 0 & 1 & 0.266 \\
\hline $\begin{array}{l}\text { Hematoma at surgical site req. reop. } \\
\text { or drainage }\end{array}$ & 11 & 3 & 8 & 0.059 \\
\hline Blood transfusion & 7 & 1 & 6 & $\mathbf{0 . 0 2 8}$ \\
\hline Pneumothorax req. chest tube & 3 & 3 & 0 & 0.119 \\
\hline Pulmonary embolism not req. surgery & 2 & 1 & 1 & 0.880 \\
\hline Vein thrombosis & 9 & 5 & 4 & 0.761 \\
\hline Vascular repair near the implant site & 1 & 1 & 0 & 0.368 \\
\hline Arrythmias & 5 & 4 & 1 & 0.071 \\
\hline Other & 2 & 1 & 1 & 0.265 \\
\hline
\end{tabular}

This article is protected by copyright. All rights reserved. 


\begin{tabular}{|l|c|c|c|c|}
\hline Total minor bleeding & 28 & 10 & 18 & $\mathbf{0 . 0 5 1}$ \\
\hline Total minor thromboembolic & 12 & 7 & 5 & 1.000 \\
\hline
\end{tabular}

Continuous variables are expressed as mean with corresponding standard deviation or median with corresponding IQR; categorical variables are expressed as absolute value with proportion between parenthesis.

Table 3. Comparison of minor complications between the "bridging" and the other two periprocedural anticoagulation strategies.

\begin{tabular}{|c|c|c|c|}
\hline & $\begin{array}{l}\text { AC interrupted \& } \\
\text { continued } \\
(\mathrm{N}=193)\end{array}$ & $\begin{array}{c}\text { AC «Bridging» } \\
\quad(\mathbf{N}=\mathbf{1 3 5})\end{array}$ & p-value \\
\hline MINOR- Total & $9(4.7)$ & $16(11.9)$ & 0.020 \\
\hline Intraprocedural & $3(1.6)$ & 0 & 0.271 \\
\hline Arrhythmia & 1 & 0 & 1.000 \\
\hline Blood transfusion & 2 & 0 & 0.514 \\
\hline Post-procedural & $6(3.1)$ & $16(11.9)$ & 0.005 \\
\hline Pericardial effusion without drainage & 0 & 1 & 0.412 \\
\hline $\begin{array}{l}\text { Hematoma at surgical site req. } \\
\text { intervention }\end{array}$ & 3 & 5 & 0.281 \\
\hline Hemotorax without chest tube & 1 & 0 & 1.000 \\
\hline Vein thrombosis & 0 & 4 & 0.028 \\
\hline Blood transfusion & 1 & 5 & 0.087 \\
\hline Pneumothorax req. chest tube & 0 & 0 & 1.000 \\
\hline $\begin{array}{l}\text { Pulmonary embolism not req. } \\
\text { surgery }\end{array}$ & 0 & 1 & 0.412 \\
\hline Other & 1 & 0 & 1.000 \\
\hline Total minor bleeding & $7(3.6)$ & $11(8.1)$ & 0.088 \\
\hline Total minor thromboembolism & 0 & $5(3.7)$ & 0.011 \\
\hline
\end{tabular}

Continuous variables are expressed as mean with corresponding standard deviation or median with corresponding IQR; categorical variables are expressed as absolute value with proportion between parenthesis.

This article is protected by copyright. All rights reserved. 